\title{
Ion Irradiation-Induced Changes in Surface Morphology of InSb
}

\author{
Alejandro Perez-Bergquist ${ }^{*}$, Xia Xiang*, Yanwen Zhang ${ }^{* * *}$, and Lumin Wang,*** \\ "Department of Nuclear Engineering and Radiological Science, ${ }^{* *}$ Department of Materials Science \\ and Engineering, University of Michigan, Ann Arbor, MI 48109 \\ ${ }^{* * *}$ Pacific Northwest National Laboratory, Richland, WA 99352
}

Ion irradiation of InSb is known to create extended void networks that result in the formation of highly porous, fiber-like InSb nanostructures [1]. Similar structures have been observed in other antimonides, such as GaSb [2], as well as other semiconductors, including germanium [3]. Although ion irradiation of other semiconductors results in the formation of porous layers with uniform, fairly flat surfaces, the irradiation of InSb with high energy ions results in the formation of porous layers with varying surface morphologies. In this study, we investigate the effects of ion energy and fluence on the surface morphology of ion irradiated InSb.

Figure 1 shows plan view SEM images of InSb samples irradiated with 1 and $3 \mathrm{MeV} \mathrm{Au}^{+}$ions with fluences ranging from $5 \times 10^{13}$ ions $/ \mathrm{cm}^{2}$ to $6 \times 10^{14}$ ions $/ \mathrm{cm}^{2}(\sim 3-35 \mathrm{dpa})$. Using $1 \mathrm{MeV}$ ions, a sample irradiated to a fluence of $1.25 \times 10^{14}$ ions $/ \mathrm{cm}^{2}$ developed a surface that resembled crumpled tissue paper (Fig. 1a). With increased ion fluence, the surface layer was gradually removed, revealing nanofibers underneath (Fig. 1b, 1c). When the ion energy was increased to $3 \mathrm{MeV}$ and a sample was irradiated to a fluence of $5 \times 10^{13}$ ions $/ \mathrm{cm}^{2}$, the surface took on a ropy, corrugated appearance (Fig. 1d). An increase in ion fluence caused the surface layer to be gradually removed and caused the features of the ropy regions to become larger but less distinct (Fig. 1e, 1f). Figure 2 clearly displays the unique bimodal surface structures that resulted from the ion irradiation process, showing that at certain ion energies and fluences there can exist both microscale ropy structures as well as nanoscale intertwining fiber-like structures.

Figure 3 shows a cross-sectional SEM image of an InSb sample irradiated with $1 \mathrm{MeV} \mathrm{Au}^{+}$ions to a fluence of $1.25 \times 10^{14}$ ions $/ \mathrm{cm}^{2}$. The figure highlights three distinct regions of the porous layer: a highly buckled yet continuous surface layer, a fiber regime where the material is composed of fairly uniformly sized and distributed InSb fibers, and a void formation regime, composed of numerous small voids embedded within a continuous InSb matrix. TEM XEDS performed on individual nanofibers showed that the fibers contained uniform concentrations of indium and antimony, and SAED of the fiber volume showed that the fibers contain both nanocrystalline and amorphous components (Fig. 4).

From analysis of SEM and AFM data, it can be seen that both ion energy and fluence impact the surface structure periodicity, size, and surface roughness. Sputtering effects tend to remove the surface layer at higher ion fluences. The exact mechanisms responsible for the development of such structures under ion beam irradiation are under investigation.

References:

[1] G. L. Destefanis and J. P. Gailliard, Appl. Phys. Lett. 36 (1980) 40.

[2] R. Callec et. al., Appl. Phys, Lett. 59 (1991) 1872.

[3] B. R. Appleton et. al., Appl. Phys. Lett. 41 (1982) 711.

[4] This study was supported by the US DOE BES with grant \#DE-FG02-02ER46005. 

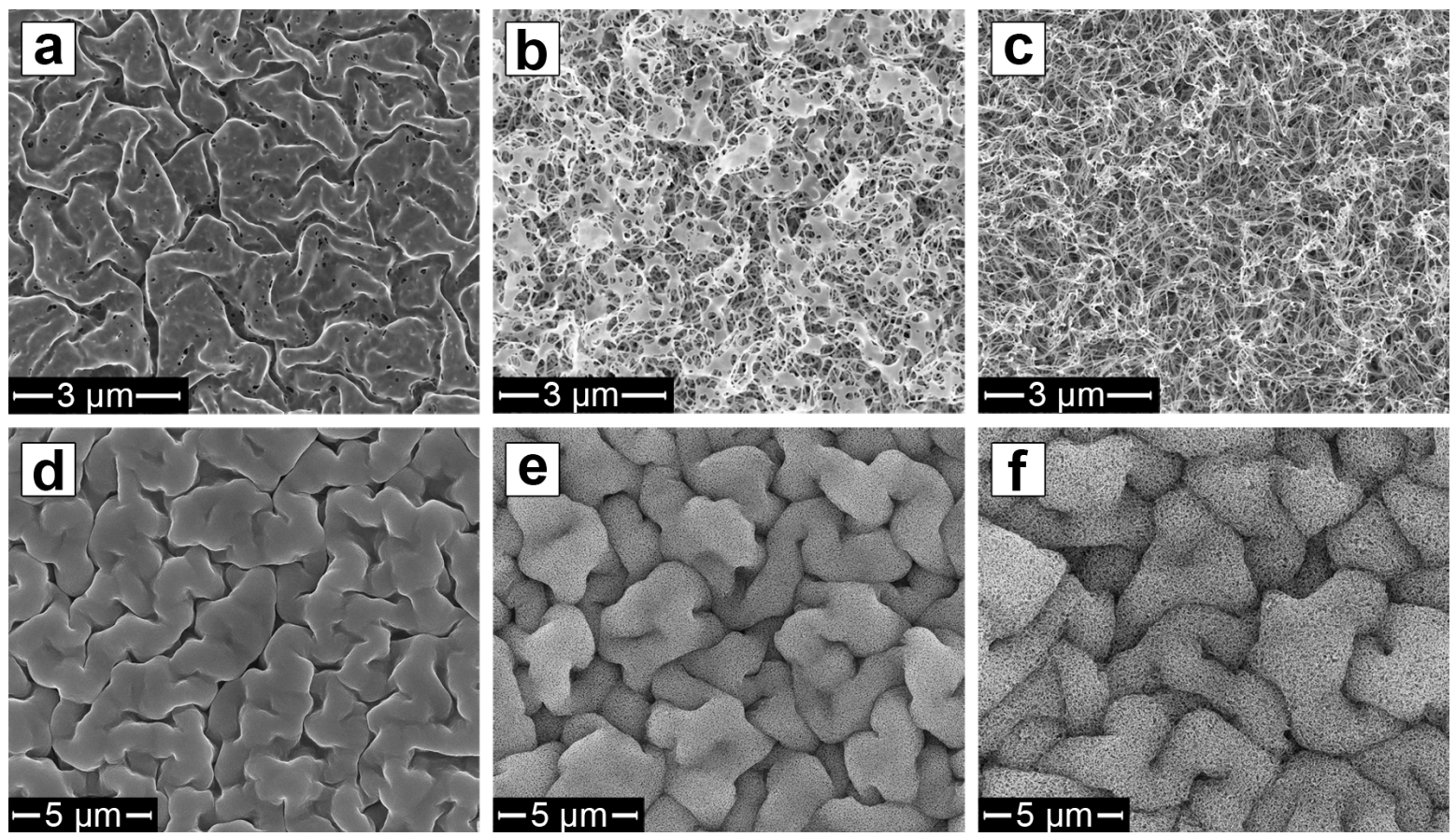

Fig. 1. Top-down SEM image of InSb samples irradiated with 1 and $3 \mathrm{MeV} \mathrm{Au}^{+}$ions. Different samples were irradiated with $1 \mathrm{MeV} \mathrm{Au}^{+}$to a) $1.25 \times 10^{14}$ ions $/ \mathrm{cm}^{2}$, b) $3 \times 10^{14} \mathrm{ions} / \mathrm{cm}^{2}$, and c) $6 \times 10^{14} \mathrm{ions} / \mathrm{cm}^{2}$ and with $3 \mathrm{MeV} \mathrm{Au}{ }^{+}$to d) $5 \times 10^{13}$ ions $/ \mathrm{cm}^{2}$, e) $1 \times 10^{14}$ ions $/ \mathrm{cm}^{2}$, and f) $2 \times 10^{14}$ ions $/ \mathrm{cm}^{2}$.

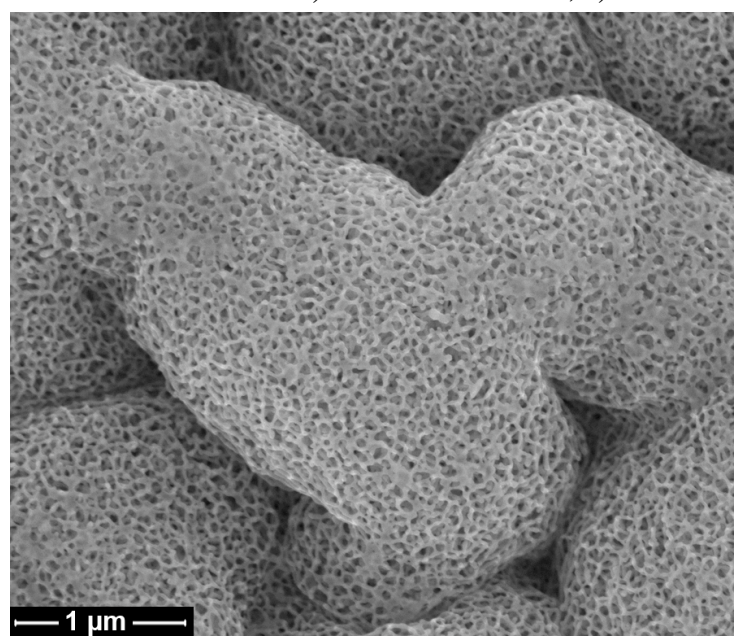

Fig. 2. Top down SEM image of an InSb sample irradiated with $3 \mathrm{MeV} \mathrm{Au}{ }^{+}$ions to $1 \times 10^{14}$ ions $/ \mathrm{cm}^{2}$. The large surface structures are roughly $2 \mu \mathrm{m}$ in diameter and are composed of nanofibers that are approximately $20 \mathrm{~nm}$ in diameter.

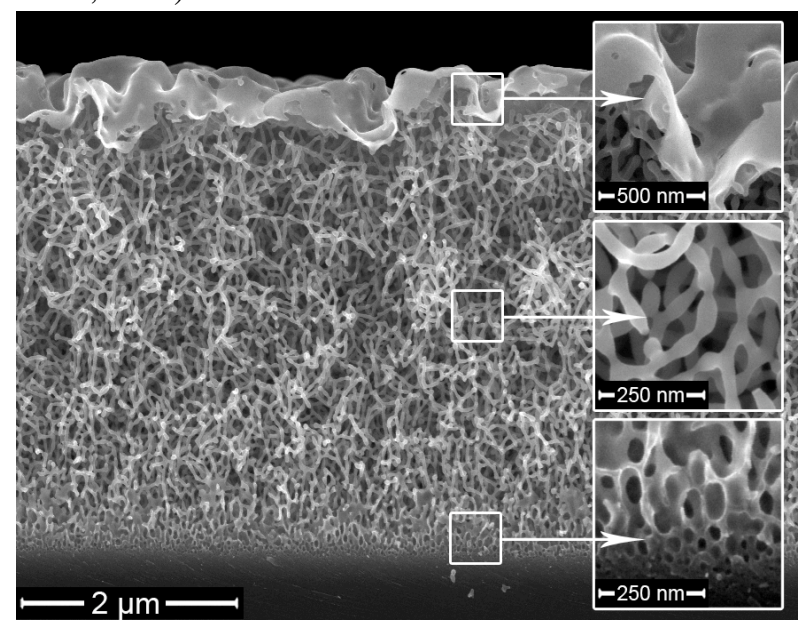

Fig. 3. Cross-sectional SEM image of an InSb sample irradiated with $1 \mathrm{MeV} \mathrm{Au}$ to a fluence of $1.25 \times 10^{14} \mathrm{ions} / \mathrm{cm}^{2}$. Three distinct regions of the porous layer are visible: a warped yet continuous surface layer, a fiber regime composed of fairly uniform InSb fibers, and a void formation regime composed of small voids embedded within a continuous InSb matrix.

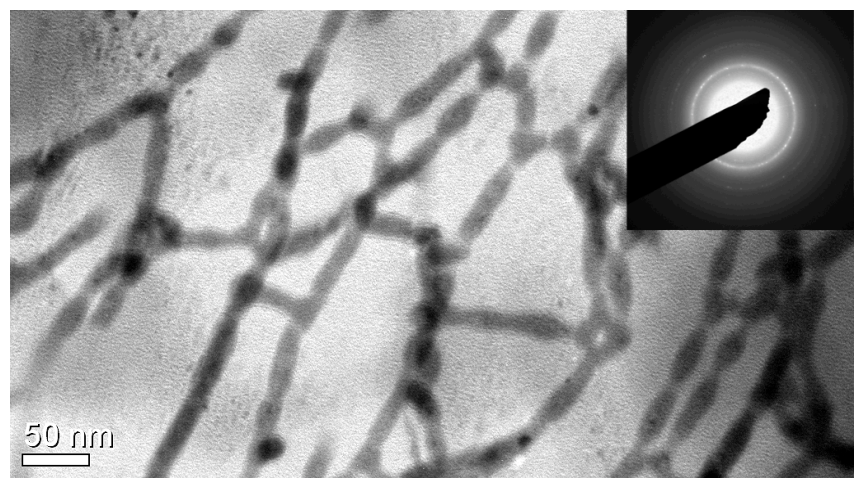

Fig. 4. BF TEM image of InSb fibers irradiated with $1 \mathrm{MeV} \mathrm{Au}^{+}$ions to a fluence of $1.25 \times 10^{14} \mathrm{ions} / \mathrm{cm}^{2}$. Insert: SAED pattern of the fiber volume, showing characteristic of amorphous and polycrystalline materials. HRTEM (not shown) confirms the presence of nanocrystalline and amorphous regions among the fibers. 